\section{A Simple Preparation Method for Holey Carbon Grids}

Bill Tivol,

Wadsworth Center and Biological Microscopy and Image Reconstruction Resource tivol@wadsworth.org

There are two distinct uses for holey carbon grids: for image checking and for support for ultrathin carbon films for cryoEM. This procedure gives holes that are too large for convenient image checking, but are the appropriate size for support of carbon films. The procedure is divided into making the formvar mixture, dipping the slides and getting the film on the grids.

I) Take $45 \mathrm{~mL}$ of chloroform, add $0.2 \mathrm{~g}$ of formvar, and shake to dissolve. A small brown glass jar with a capacity of $100 \mathrm{~mL}$ is the best container to use, and the jars from commercial formvar solution are ideal.

2) Add $45 \mathrm{~mL}$ of acetone and $1 \mathrm{~mL} 90 \%$ glycerine and shake vigorously for about ten minutes. Sonicate the mixture for 5 minutes with a probe sonicator: the strength of sonication is important. If the mixture is used immediately, I get a very large number of small holes about $2 \mu \mathrm{m}$ and if the mixture stands for about 1 hour, I get a large number of larger holes, about $5 \mu \mathrm{m}$. If the mixture has not been used within several hours, it can be resonicated. I almost always get "wall-to-wall" holes with very thin bars of formvar remaining.

3) The slides should be prepared by whatever method allows the formvar to be stripped from their surfaces. This seems to vary from lab to lab, so use what works for you. Fill a $50 \mathrm{~mL}$ beaker with the formvar mixture. Lower the slide two to three $\mathrm{cm}$ into the mixture, and hold it there for five seconds or longer. Slowly withdraw the slide at an angle; the side forming an acute angle with the formvar mixture will be the better one to use. Dip the slide into a beaker of acetone past the level of the formvar film, and agitate it gently, leaving it immersed for about five seconds. Withdraw the slide, and stand it on end until the solvents evaporate. Check the slide with a phase contrast microscope. You should see smooth, round holes with high contrast. If the contrast is low, the holes have not penetrated all the way through the formvar, and the slide must be immersed in acetone again. Lower the slide at a shallow angle into a staining dish filled with warm distilled water; the better side (see last paragraph) should be up.

4) Lay grids on the film and pick up the film-and-grid assembly with Parafllm. Let dry, then coat with a very thick layer of carbon, about 80 to $100 \mathrm{~nm}$, which will look dark gray on a white material, such as filter paper.

S) Remove the grids from the Parafilm and place them carbon side up on a piece of filter paper in a glass petri dish. Carefully pour chloroform onto the filter paper so that it is saturated. Do not float any of the grids, this can result in pile-up and will weaken the carbon nets. Cover the dish and place in a fume hood overnight. Note that it is difficult to add the chloroform while the petri dish is in the hood due to the air flow -- if this is not the case, you need more air flow in your hood.

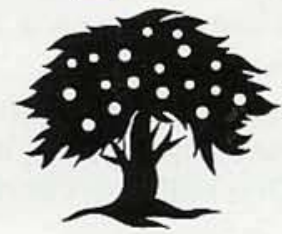

\title{
$609-252-9192$
}

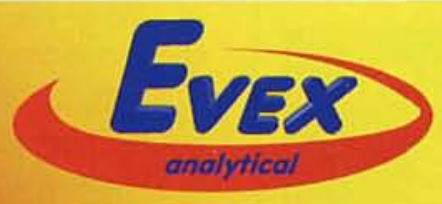

WWw.evex,com

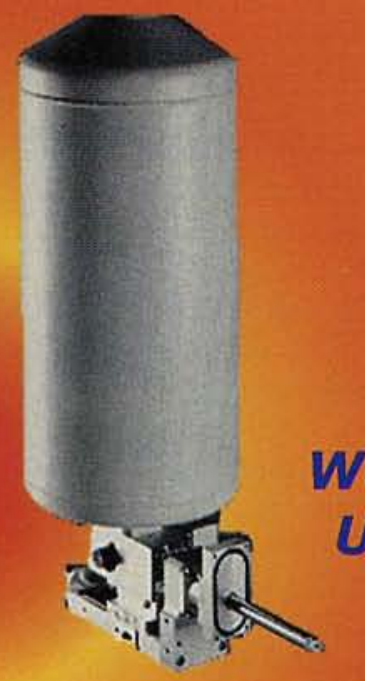

\section{Universal Pulse Processor}

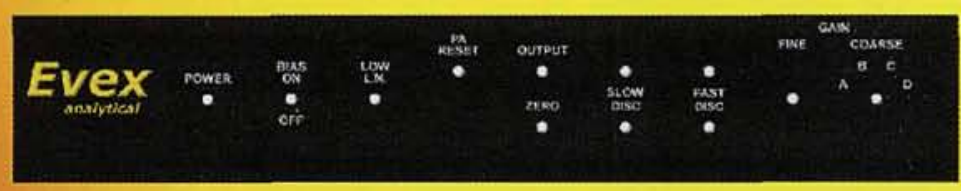

We Will Service

any

X-ray Analyzer Nationwide

\section{X-ray Microanalysis}

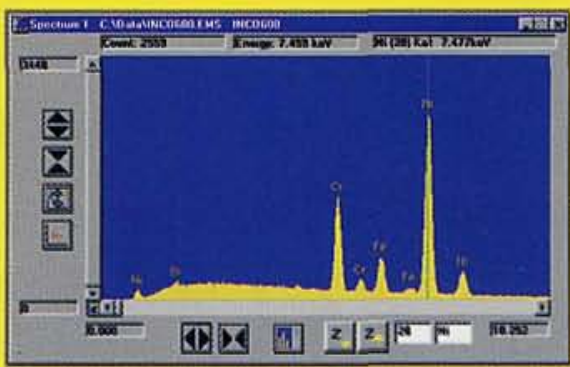

